Check for updates

Cite this: J. Mater. Chem. C, 2017, 5,5487

Received 23rd January 2017,

Accepted 2nd May 2017

DOI: $10.1039 / \mathrm{ctc00392g}$

rsc.li/materials-c

\section{UV-curable hybrid organic-inorganic composite inks with a high refractive index for printing interference images and holograms}

\author{
Elena A. Eremeeva, (D)*a Aleksandr V. Yakovlev, (D) ${ }^{a}$ Evgeny A. Pidko (D) ${ }^{\text {ab }}$ and \\ Alexandr V. Vinogradov*a
}

\begin{abstract}
Herein we report a new, facile and inexpensive methodology for obtaining highly refractive polymers suitable for inkjet printing using hexacoordinated titanium complexes (THC) and a UV-curable lacquer based on triethylene glycol dimethacrylate (TGD) that is a cheap and non-toxic monomer that can be rapidly polymerized under UV irradiation under ambient conditions. The resulting polymeric materials are characterized by a refractive index (RI) of about 1.85 in the solid state and have rheological properties suitable for inkjet printing applications. The polymeric materials can be printed onto solid supports resulting in surface structures giving rise to interference images and masking of rainbow holograms illustrating thus the prospective practical applications of the described approach.
\end{abstract}

\section{Introduction}

The development of advanced liquid-phase printing methods capable of depositing inks with a resolution of as low as $1 \mu \mathrm{m}$ holds promise to pave the way towards versatile and readily available technologies for the production of optical nanostructures and nanodevices. Techniques based on inkjet printing of functional inks have been extensively discussed in the recent literature as practical approaches to directly pattern various classes of materials on supports for applications in electronics, ${ }^{1,2}$ micromechanics, ${ }^{3}$ biomedicine ${ }^{4}$ and other areas. ${ }^{5-7}$ In our previous studies, we have reported the development of functional inorganic inks for inkjet printing of well-defined heterostructures suitable for applications such as color interference images ${ }^{8}$ and masking rainbow holograms. ${ }^{9}$

Conventionally, the spatial resolution in inkjet printing of functional materials is limited to $10-20 \mu \mathrm{m}$ as determined by a combination of factors such as the droplet diameters, placement errors and physicochemical characteristics of the inks. The printing resolution can be substantially improved up to $<10 \mu \mathrm{m}$ by using for example electrohydrodynamic jet printing methods, in which the inks are deposited onto the substrates as uniform droplets of ultra-low volumes created by flows controlled by electric fields rather than thermal or acoustic energy. Recent studies demonstrate the potential of this methodology for the precise printing of

\footnotetext{
${ }^{a}$ ITMO University, Lomonosova St., 9, Saint Petersburg, 192001, Russia.

E-mail: eremeeva@scamt.ru,avv@scamt.ru

${ }^{b}$ Inorganic Materials Chemistry group, Eindhoven University of Technology,

P.O. Box 513, 5600MB, Eindhoven, The Netherlands
}

electronic devices at a submicron resolution. ${ }^{9-11}$ An important limitation of this approach is, besides the rather low printing speed, the charging of the droplets that might be particularly devastating when metastable inorganic or hybrid inks are employed. Potentially the printing resolution of several $\mu \mathrm{m}$ could be achieved by using aerosol jet liquid-phase printing, ${ }^{12}$ in which a dense aerosol of droplets with a size of 1 to $5 \mu \mathrm{m}$ is utilized to create a focused ink beam suitable for high-resolution patterning of a wide range of functional liquids including nanoparticle suspensions, precursors and biological materials. ${ }^{13-18}$

In the context of optical devices, the applicability of the aerosol-jet technique is usually limited by a variety of crucial factors related to the optical and mechanical characteristics of the printed structures. A common strategy for the adjustment of the rheological properties of inks to make them suitable for printing involves the utilization of organic additives, which makes it literally impossible to achieve high refraction indices (RIs) in a solid deposited layer. The most common additives such as perfluorooctanesulfonic acid (PFOS) used to reduce surface tension and ethylene glycol commonly employed as a viscosity regulator have RIs of $c a .1 .46$ and 1.43, respectively. The utilization of colloidal inks based on inorganic sol-gel materials represents one of the most promising practical approaches for printing optical nano- and heterostructures with high RIs. ${ }^{19}$ The practical application of such methodologies in the fabrication of devices such as LEDs, planar waveguides and diffraction gratings is limited due to the poor mechanical properties and insufficient uniformity of the surface structures due to the limited control over the condensation processes in the pure inorganic matrices. As a result, this technological field is 
dominated by much more costly techniques based on hightemperature annealing.

Herein we propose that these fundamental limitations can be addressed by making use of hybrid UV-hardening organicinorganic inks. Such an approach would potentially offer better control over the condensation process on the surface and, ultimately, over the morphology and optical characteristics of the final printed patterns. We present a new methodology for the production of UV-hardening polymers with a high refractive index based on complexes of oligoesteracrylates with hexacoordinated Ti(Iv) complexes.

Previous studies have discussed the preparation of transparent optical polymers with increased optical density, typically comprising various functionalized moieties such as aromatic groups, halogens, amines or sulfur-containing functionalities as well as metals integrated within the polymer itself. ${ }^{20}$ An alternative approach is based on doping of organic polymeric matrices with nanoparticles made of highly refractive substances such as $\mathrm{TiO}_{2}, \mathrm{ZnS}, \mathrm{CeO}_{2}, \mathrm{ZrO}_{2}, \mathrm{ZnO}, \mathrm{Bi}_{4} \mathrm{Ti}_{3} \mathrm{O}_{12}$, etc. ${ }^{21-26,31}$ However, the resulting composite materials usually exhibit high heterogeneity resulting in strong light scattering. ${ }^{14}$ They also typically show limited stability in water and air. To overcome these limitations, we made use of the established sol-gel chemistry approaches based on the coordination chemistry of titanium isopropoxide. ${ }^{26-30}$ We prepared and optimized the rheological properties of hybrid inorganic-organic UV-hardening inks, which were then successfully employed for efficient high-resolution printing of highly refractive elements on solid supports.

\section{Experimental}

\section{Chemicals}

Titanium tetraisopropoxide TTIP $\left(\mathrm{Ti}\left(\mathrm{O}^{\mathrm{i}} \mathrm{Pr}\right)_{4}\right.$, Sigma-Aldrich, 98\%), glacial acetic acid $\left(\mathrm{CH}_{3} \mathrm{COOH}\right.$, Sigma-Aldrich, 99.9\%), and UV-curable lacquer based on triethylene glycol dimethacrylate (TGD, Aldrich, 99\%) were used as received for the synthesis.

UV-curable polymers were prepared according to the following procedure. Titanium tetraisopropoxide was added to $\mathrm{CH}_{3} \mathrm{COOH}$ in a molar ratio of $1: 1$ under continuous stirring until a transparent yellow liquid was formed. The reaction with the acetic acid led to a change in the coordination of the $\mathrm{Ti}^{4+}$ ions from tetrahedral to a more stable octahedral configuration and the reaction of polycondensation with removing isopropanol alcohol as a product resulting in the formation of clustered species, as illustrated in Scheme 1. The reaction was exothermic and accompanied by substantial warming up of the reaction vessel. After cooling, $1 \mathrm{ml}$ of TGD was added to $6 \mathrm{ml}$ of the reaction mixture under ambient conditions and continuous stirring. This ratio was selected based on our extensive preliminary experiments that demonstrated that this ratio enables maximizing the refractive index and at the same time avoiding the undesirable effects due to segregation and cracking upon the condensation of the solid phase. The mixture was homogenized for a day, after which gradual colouring of the solution to yield a bright yellow colour was observed (Fig. 1).

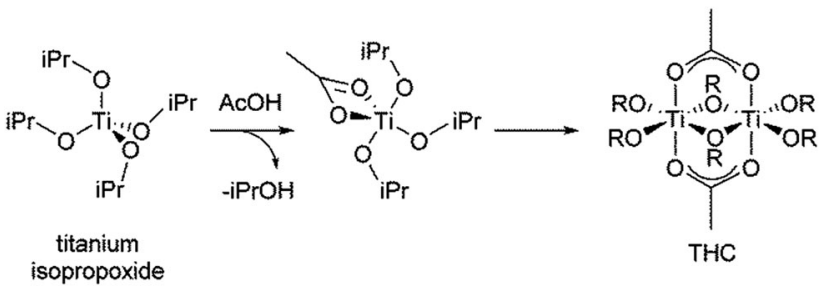

Scheme 1 The formation of the THC adduct by the reaction of titanium isopropoxide with acetic acid.

The interaction of the THC with TGD oligoesteracrylate proceeds via replacing the residual isopropyl groups with acrylate moieties most likely driven by the formation of stable 5-membered ring metallacycle moieties that increase the overall stability of the complex and makes it insensitive to moisture (Scheme 1).

Curing of the polymeric films was performed using a BLE-125B/ C (125 W) UV lamp by exposing the films to UV light for 5 minutes under ambient conditions. The radical polymerization of the THC-TGD adduct was initiated by UV light and after hardening yielded uniform solid structures with a high refractive index.

\section{Ink preparation}

The THC-TGD adduct was used as a base for the inks, whose viscosity was adjusted by adding isopropanol to achieve a good printability in UV three-dimensional printing. The viscosity parameters were studied using a Fungilab EXPERT L rotary viscometer. The measurements were carried out using an L1 spindle at a rotation speed of $100 \mathrm{rpm}$. The maximum achieved value of the sample viscosity was $60 \mathrm{mPa}$. The inks were tested using a Roland LEF-12 UV printer.



Fig. 1 Visual characteristics of the UV-curable ink with a high refractive index and its principal components (A) the THC compound; $(B)$ triethylene glycol dimethacrylate; $(C)$ the as-synthesized lacquer with a high refractive index. 
The refractive index of the thin films of a highly refractive UV-curable lacquer was assessed by optical reflection measurements at normal incidence. Non-polarized light from a halogen lamp (HL-2000-FHSA) was focused on the film surface by a lens (Mitutoyo MPlanAPO, NA 0.55) capable of $50 \times$ magnification. The reflected light was collected by the same lens and redirected to a spectrometer (HORIBA Lab Ram HR) with a cooling CCD camera (AndorDU 420A-OE) and a diffraction grating with a period of $150 \mathrm{~g} \mathrm{~mm}^{-1}$. The resulting spectrum was normalized by the known spectrum of the halogen lamp. The reflection spectrum of the different areas of the films allowed us to estimate an error in the refractive index at different wavelengths. Based on the interference spectrum, the refractive index of the films was calculated according to an example shown in ref. 8.

Powder X-ray diffraction analysis was carried out using a Bruker D8 Advance diffractometer. Standard procedures were employed for the preparation of the formulations. The diffractograms were collected using CuK $\alpha 1$ irradiation with a nickel filter, at a voltage and a tube current of $35 \mathrm{kV}$ and $20-22 \mathrm{~mA}$, respectively, a counter movement rate of $1^{\circ} \mathrm{min}^{-1}$, a chart tape rate of $2400 \mathrm{~mm}$ hour $^{-1}$ and a count rate of 200-1000 pulses per s with a time constant of $5 \mathrm{~s}$.

Crystalline germanium was used as the internal standard. The diffractograms were recorded in the $2 \theta$ range of $5-75^{\circ}$. The positions of the maxima were measured from peak heights assuming that they correspond to a wavelength $\lambda_{\alpha 1}=1.54051 \AA$.

Fourier transform infrared (FTIR) spectroscopy was used to analyze three samples, namely, the THC, oligoesteracrylate - TGD, and the highly refractive UV-curable lacquer derived from them. The measurements were conducted in the transmission mode and the spectra were collected in the wavelength range of 500-4000 nm under ambient conditions $\left(T=26^{\circ} \mathrm{C}\right.$, dilution sample : isopropanol $=$ $1: 1000)$ using a Shimadzu IRAffinity- 1 spectrometer with a singlebounce ATR accessory with a Ge prism. The baseline was recorded for isopropanol that was also used as a solvent for all samples.

UV-vis spectrometry analysis of nanocomposite films on a quartz glass surface was performed using an Agilent Cary $60 \mathrm{UV}$ spectrophotometer in the wavelength range of $200-600 \mathrm{~nm}$ in the transmission mode under ambient conditions (room temperature and atmospheric pressure). The spectrum of the isopropanol solvent was used as for the background correction.

Scanning electron microscopy (SEM) was employed to investigate the morphological properties of surface structures using a Tescan Vega 3 scanning electron microscope. Sample preparation involved the deposition of the highly refractive UV-curable lacquer sample on a silicon substrate and drying under an UV lamp.

The analysis of the surface topography of the applied layer of the highly refractive lacquer polymerized by exposure to ultraviolet light after embossing a rainbow holography pattern was carried out on a Solver NEXT atomic force microscope in tapping mode.

\section{Results and discussion}

A careful selection of suitable precursors and methodologies for the production of highly refractive lacquers is necessary to achieve maximal absorbance of the hardening material.

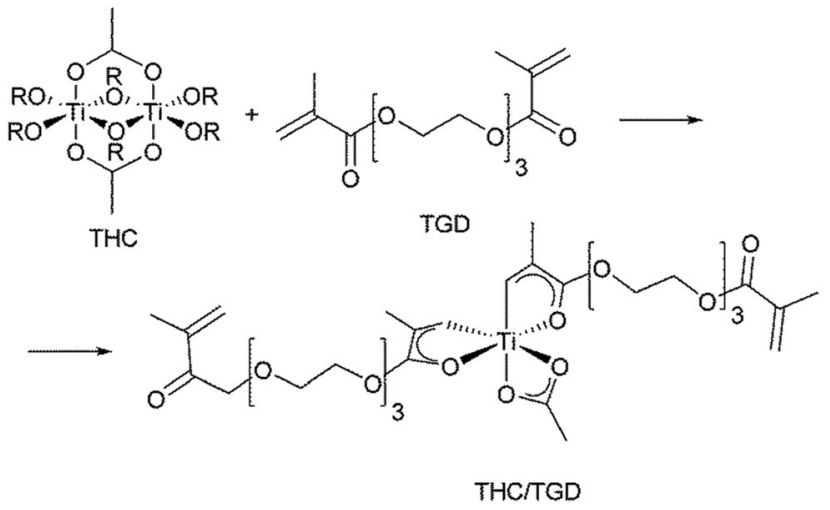

Scheme 2 The interaction of oligoesteracrylate with the THC.

The key challenge here is to achieve an optimal balance between the concentrations of the UV lacquer and the transition metal complexes. An excess of the transparent coordination compounds, usually alkoxides, reduces the lacquer stability in air due to incomplete replacement of alkoxide groups, and an excess of lacquer prevents from achieving high values of refractive indexes. In our studies, we used titanium alkoxide, whose coordination properties can be adjusted by the reaction with carboxylic acid to yield hexacoordinated oligonuclear species (Scheme 1). Previous studies gave reasons to believe that the formation of such a structure does not allow for the replacement of more than two isopropoxide groups at the titanium center. ${ }^{7,8}$ The condensation of the chelate complexes formed at the initial stage of the reaction produce polynuclear complexes bearing acetate and isopropoxide groups. Because of the high basicity of the residual titanium isopropoxide moieties, the resulting adducts still exhibit quite a poor stability under ambient conditions and undergo hydrolysis by reacting with moisture in air.

The introduction of the organic oligoesteracrylate (TGD) matrix provides chelating moieties capable of eliminating the isopropoxide species into the second coordination sphere of $\mathrm{Ti}$ so that the subsequent hydrolysis would not affect the principle structure of the complexes. The plausible reaction scheme for such a transformation is illustrated in Scheme 2.

The formation of the chelating agent with the conjugated $\pi$-system of TGD is indirectly evidenced by the enhancement of color of the TGD/THC adduct (Fig. 1).

The complexation between the TGD and THC moieties as well as the formation of the UV-curable links is further confirmed by the FTIR spectroscopic data summarized in Fig. 2. The bands at 650,990 and $1660 \mathrm{~cm}^{-1}$ in the FTIR spectrum of TGD are characteristic of the vibrations of the terminal $=\mathrm{CH}_{2}$ groups. These bands are eliminated when the HRI UV-hardening lacquer is formed indicating the reaction of these moieties with the $\mathrm{Ti}$ centers upon complexation of TGD with THC (black spectrum in Fig. 2). The weak band at $1631 \mathrm{~cm}^{-1}$ corresponds to either traces of water confined in the lacquer matrix or unreacted methylene moieties. The bands at 1569 and $1470 \mathrm{~cm}^{-1}$ in the spectra of THC and the HRI UV-hardening lacquer are attributed to the asymmetric and symmetric stretching vibrations of the carboxylic moieties 


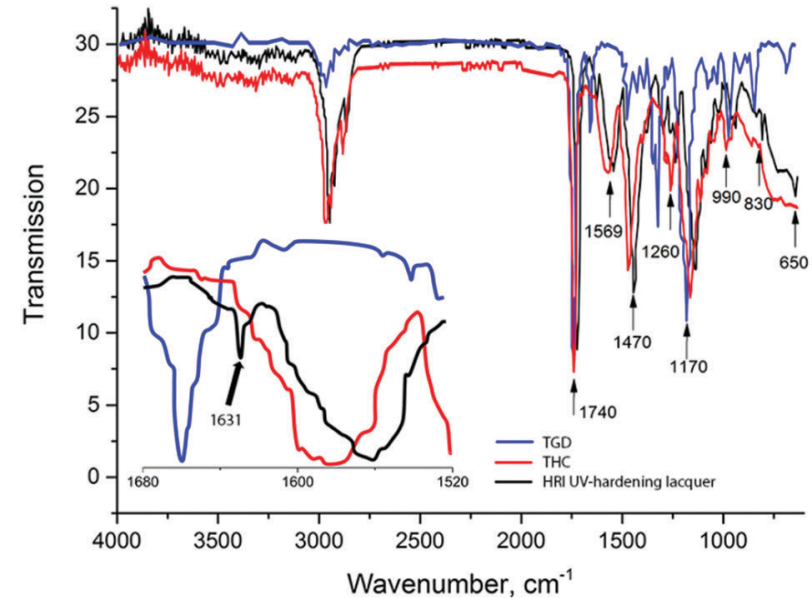

Fig. 2 FTIR transmittance spectra of the as-synthesized samples: TGD (blue line), THC (red line), and the HRI UV-curable ink (black line).

forming a bidentate coordination with Ti, while the monodentate carboxyl moieties give rise to the bands at 1260 and $1740 \mathrm{~cm}^{-1} \cdot 32$ The bands in the region $1150-1170 \mathrm{~cm}^{-1}$ most likely correspond to a superposition of the vibrational modes due to various Ti-O-C and $\mathrm{C}-\mathrm{O}-\mathrm{C}$ moieties.

A high degree of amorphization of the UV-curable lacquer in its solid state is confirmed by the powder X-ray diffraction analysis (XRD) results shown in Fig. 3. The absence of reflexes due to the crystalline titanium dioxide phase indirectly confirms our hypothesis on the lack of extensive hydrolysis of Ti species in the system. The feature at around $17^{\circ} 2 \theta$ in the XRD pattern is most likely due to the polynuclear oxotitanic species. ${ }^{33}$

Fig. 4 shows a comparison of the main optical characteristics of the highly refractive polymer and the starting materials. TGD, THC substrates and TTIP are completely transparent in the visible region of the spectrum.

The spectra of the Ti complexes (TTIP and THC) are characterized by a strong absorption below $320 \mathrm{~nm}$, whereas that of the TGD shows a distinct feature with a maximum at $350 \mathrm{~nm}$.

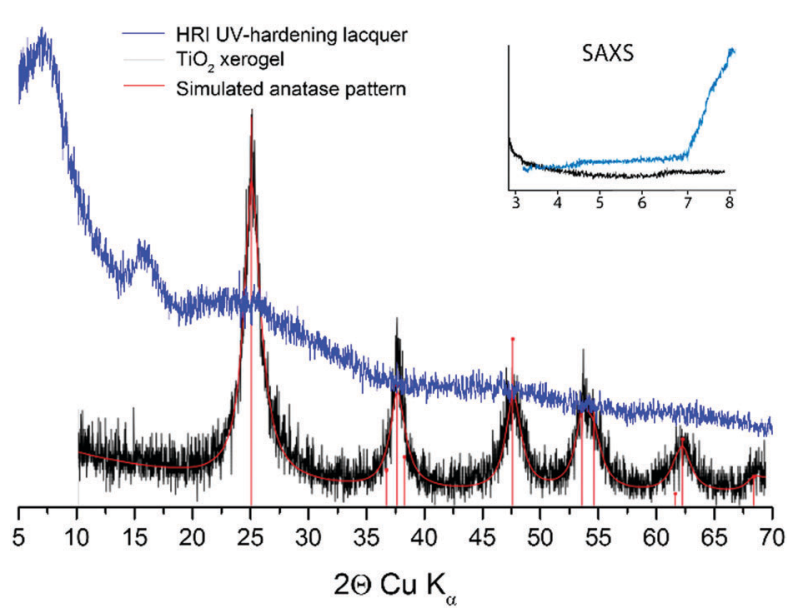

Fig. 3 X-Ray diffraction pattern of the as-synthesized HRI UV-curable lacquer after solidification in UV light (blue line) in comparison with the patterns for the nanocrystalline titania prepared in aqueous media (black line).


Fig. 4 (A) UV-vis absorption spectrum of the initial polymer with the refractive index of $T G D$, produced a highly refractive lacquer capable of UV curing, TTIP and THC; (B) dispersion of refractive indexes of the UV-curable printing ink in its cured state compared to the RI data for a thin film of titanium dioxide and a cured thin film of TGD. The refractive indices of films were obtained with use of the equation $R(\lambda)=\left[\frac{n_{1} \cdot n_{3}(\lambda)-n_{2}^{2}(\lambda)}{n_{1} \cdot n_{3}(\lambda)+n_{2}^{2}(\lambda)}\right]^{2}$, where $n_{1}=1$ is the refractive index of air, $n_{3}(\lambda)$ is the refractive index of fused glass and $n_{2}$ is the RI of the highly refractive polymer. (C) The influence of the chemical composition of the UV-curable ink (THC contents) on the reactive index.

The complexation of THC with TGD results in a substantial red shift of the main absorption peak by $c a$. $30 \mathrm{~nm}$. These spectral changes clearly indicate the formation of a direct interaction between the components and the replacement of the small ligands with the oligomeric unsaturated species. Such interaction with a ratio of 1:6 made it possible to achieve a refractive index of about 1.85 , which can be considered very high for UV-hardening compounds available to date. 
The RIs of a pure $\mathrm{TiO}_{2}$ xerogel containing 95\% nanocrystalline titanium dioxide phase ${ }^{8}$ (Fig. 3 and 4) and the current hybrid lacquer differ by only $7 \%$ (Fig. 4B). Fig. 4C shows the dependence of the refractive index on the volume ratio of THC to TGD phases. The results clearly show the uniform increase in the refractive index with increasing TGD concentration that stabilizes at a value of $c a$. 1:6 after which the RI remains constant despite the increase in the concentration of TGD.

Bearing in mind the prospective utilization of the synthesized TGD/THC adducts as UV-hardening inks, we further carried out additional experiments aimed at optimizing their rheological properties towards optimal printability. Two main parameters were varied during the optimization: (1) the RI of the inks by controlling the interaction between the THC and TGD and (2) the viscosity. The two parameters were interconnected as the TGD/THC ratio had a profound impact on the viscosity (Fig. 5A). Here, we targeted a printing-ready viscosity of the final ink of $20 \mathrm{cP}$. The parameter was adjusted by dilution with isopropyl alcohol (Fig. 5B). The optimal ratio in the system between HRI UV curable lacuer and $\mathrm{PrOH}$ (with the minimal iPrOH concentration) resulting in the optimal viscosity was obtained at the $2: 1$ volume ratio. Such a composition of the ink made it possible to achieve high-speed printing with UV fixation in thin layers.
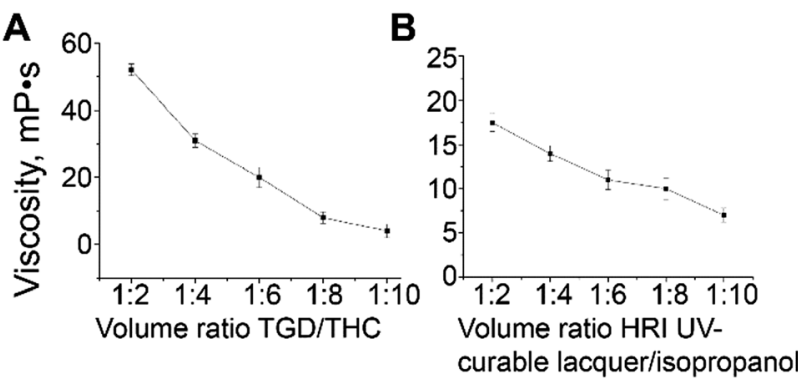

Fig. 5 The variation of the viscosity of the hybrid ink solution as a function of (A) the volume ratio between the TGD oligoesteracrylate and the THC titanium complex precursor and (B) the dilution with isopropanol.

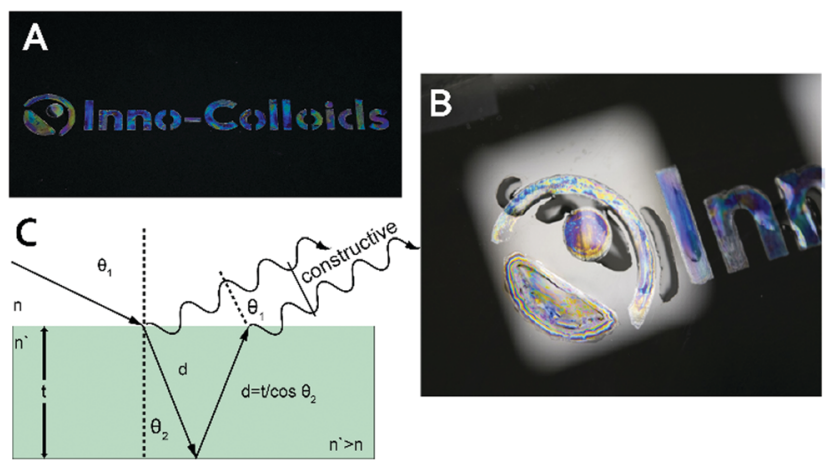

Fig. 6 The photographs of (A and B) an inscription printed using the UV-curable ink with an applied drop of water. (C) Scheme of the emergence interference in the thin film. $\theta_{1}$ - angle of incidence; $\theta_{2}$ - refraction angle; $n, n^{\prime}$ - refractive indexes of environments; $t$ - thickness of the film; $d$ - path difference of reflection light beams.
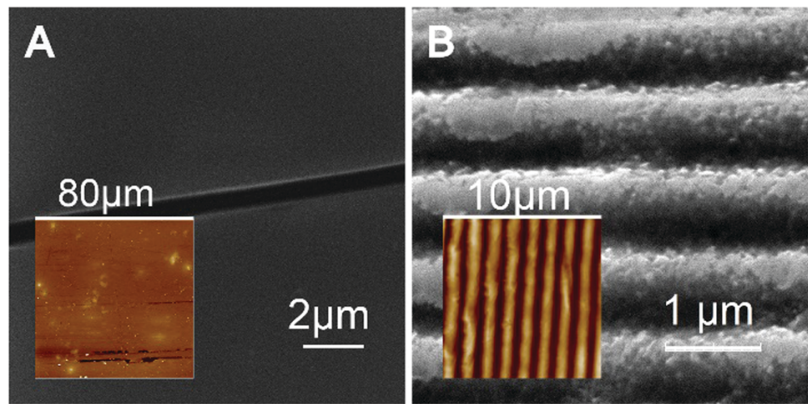

Fig. 7 SEM microimages of printed patterns of the (A) interfering layer on the glass slide and (B) printed mask on rainbow holograms, with AFM topologies shown in the inset.

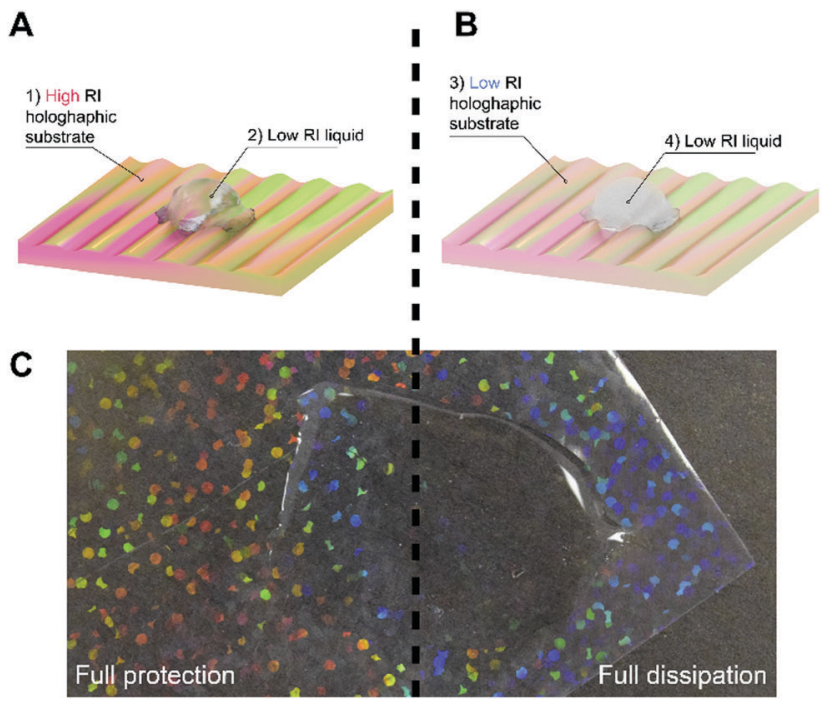

Fig. 8 (A) Model of light passing through a liquid with a low RI reflected from the microembossed lowly refractive surface of the substrate with complete loss of the diffraction effect; (B) visualization of light passing through a liquid with a low RI reflected from the microembossed highly refractive surface of the substrate while maintaining the diffraction effect: (C) the photograph of a drop of water on the holographic film surface: left part - protected with the HRI lacquer, right part - without any coating.

The synthesized ink was further tested for producing interference images (Fig. 6-8). The latter were produced by the method of microembossing followed by UV curing. Besides the potential application in the inkjet technology and offset printing, the main practical significance of the current results is that we were able to achieve a high RI for the deposited layer along with high stability. For example, microembossed rainbow holograms based on conventional polymeric materials such as polycarbonates, polyethylene, polyethylene terephthalates and other organic macromolecules disappear with time. The adsorption of organic acids and water on its surface leads to complete elimination of the diffraction effect (Fig. 8).

The patterns formed using the current hybrid organicinorganic inks retain their high RI independent of the presence of any organic phase on the surface and also show a high stability of the optical characteristics with time (Fig. 4). Attenuation of a rainbow hologram under a layer of substances 

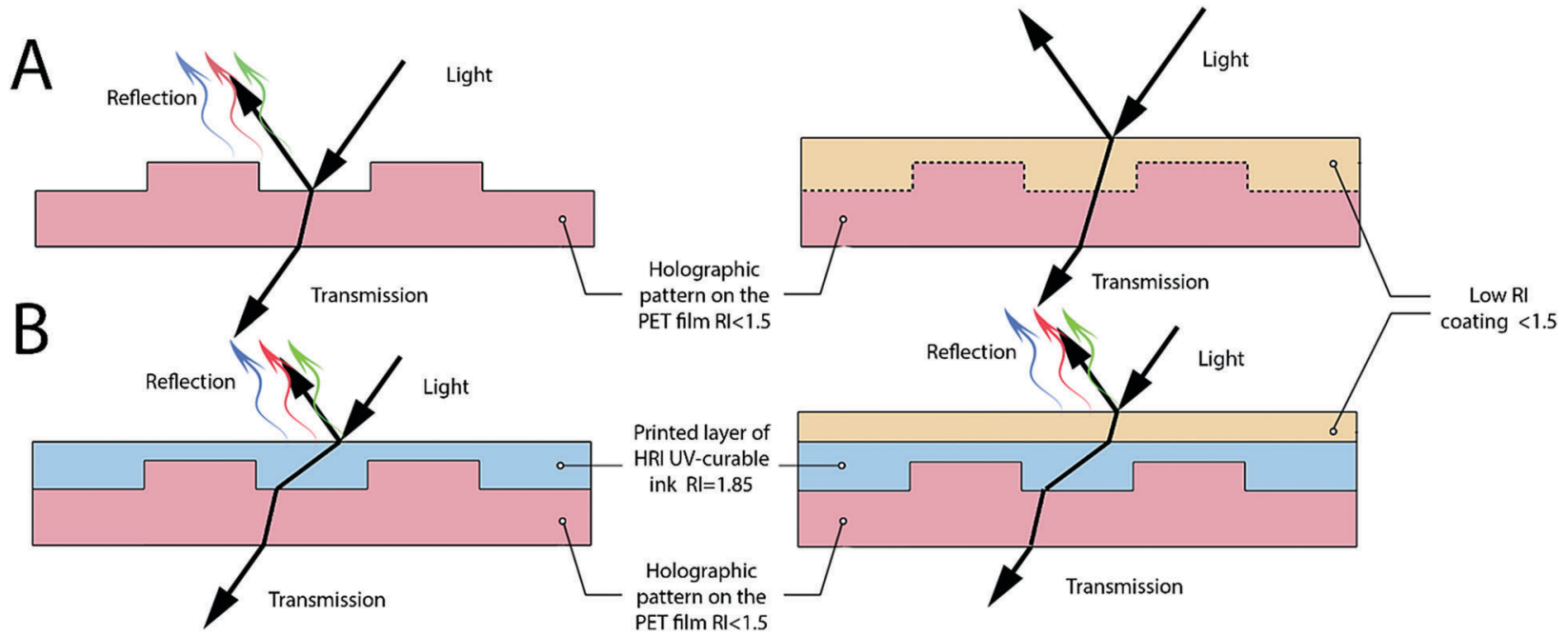

Fig. 9 (A) Scheme of light passing through a structure with a low refractive index; (B) scheme of light passing through a protected structure by inkjet printing using the HRI UV-curable ink.

with a low refractive index is shown in Fig. 6 and 8. It is clearly seen that the microembossed relief pattern hides behind the mask layer with a low RI and the passing beam of light penetrates through the structure without reflection from the surface of the film, because there is no clear boundary between the two media with different refractive indexes.

Fig. 9 illustrates schematically the mechanism by which the light beam passing through a microembossed material with a high RI and a layer with a low RI applied on it. In this case, the loss of the rainbow holographic pattern is not observed, because the passing beam of light is refracted and reflected from the microordered structure, which retains the rainbow image on the film.

\section{Conclusions}

We have developed a method for obtaining UV-hardening printing inks with a refractive index as high as 1.85 in the entire visible range. The interaction of a $\mathrm{Ti}^{4+} \mathrm{THC}$ with TGD, followed by the modification of the rheological properties by dilution with isopropanol as a modifying agent allowed achieving viscosity $(16.8 \mathrm{cP})$ suitable for inkjet printing applications. The reported method makes it possible to manufacture optical elements with high optical uniformity and transparency, as well as improved mechanical characteristics. We propose that this method can be utilized in the future for printing planar waveguides, producing rainbow holograms, and forming photon-induced panels.

\section{Competing financial interest}

The authors declare no competing financial interest.

\section{Acknowledgements}

The authors are grateful to Prof. David Avnir for comprehensive support of the SCAMT lab and scientific advice. We gratefully acknowledge financial support from the Russian Scientific Foundation (grant no. 16-19-10346).

\section{References}

1 Z. P. Yin, Y. A. Huang and N. B. Bu, et al., Chin. Sci. Bull., 2010, 55, 3383-3407.

2 B. Derby, Annu. Rev. Mater. Res., 2010, 40, 395-414.

3 S. Nakano, T. Sekitani, T. Yokota and T. Someya, Appl. Phys. Lett., 2008, 92, 053302.

4 S. M. Safaryan, A. V. Yakovlev, E. A. Pidko, A. V. Vinogradov and V. V. Vinogradov, J. Mater. Chem. B, 2017, 5, 85-91.

5 P. Gao, A. Hunter, S. Benavides, M. J. Summe, F. Gao and W. A. Phillip, ACS Appl. Mater. Interfaces, 2016, 8, 3386-3395.

6 B. Lorbera, W.-K. Hsiaoc and K. R. Martin, Curr. Opin. Ophthalmol., 2016, 27(3), 262-267.

7 A. V. S. Parry, A. J. Straub, E. M. Villar-Alvarez, T. Phuengphol, J. E. R. Nicoll, X. L. W. K., L. M. Jordan, K. L. Moore, P. Taboada, S. G. Yeates and S. Edmondson, J. Am. Chem. Soc., 2016, 138(29), 9009-9012.

8 A. V. Yakovlev, V. A. Milichko, V. V. Vinogradov and A. V. Vinogradov, ACS Nano, 2016, 10, 3078-3086.

9 A. V. Yakovlev, V. A. Milichko, V. V. Vinogradov and A. V. Vinogradov, Adv. Funct. Mater., 2015, 25(43), 7375-7380.

10 J.-U. Park, M. Hardy, S. Jun Kang, K. Barton, K. Adair, D. Mukhopadhyay, C. Young Lee, M. S. Strano, A. Alleyne, J. G. Georgiadis, P. Ferreira and J. Roger, Nat. Mater., 2007, 6, 782-789.

11 AEROSOL JET ${ }^{\circledR}$ PRINTED ELECTRONICS OVERVIEW.

12 B. Kahn, Org Printed Electron, 2007, vol. 1, pp. 14-17.

13 K. Hong, S. H. Kim, A. Mahajan and C. Daniel Frisbie, ACS Appl. Mater. Interfaces, 2014, 6(21), 18704-18711.

14 M. Ha, J.-W. T. Seo, P. L. Prabhumirashi, W. Zhang, M. L. Geier, M. J. Renn, C. H. Kim, M. C. Hersam and C. Daniel Frisbie, Nano Lett., 2013, 13(3), 954-960. 
15 A. Mette, P. L. Richter, M. Hörteis and S. W. Glunz, Prog. Photovoltaics, 2007, 15, 621-627, DOI: 10.1002/ pip.759.

16 I. Grunwald, et al., Biofabrication, 2010, 2, 014106.

17 R. Liu, et al., Nanotechnology, 2012, 23, 505301.

18 M. L. Brongersma, Y. Cui and S. Fan, Nat. Mater., 2014, 13, 451-460.

19 T. Higashihara and M. Ueda, Macromolecules, 2015, 48(7), 1915-1929.

20 T. Asai, W. Sakamoto and T. Yogo, J. Nanopart. Res., 2012, 14, 1262, DOI: 10.1007/s11051-012-1262-1.

21 C. Lü, Z. Cui, Y. Wang, Z. Li, C. Guan, B. Yang and J. Shena, J. Mater. Chem., 2003, 13, 2189-2195.

22 Z. C. Orel and B. Orel, Phys. Status Solidi B, 1994, 186, K33-K36, DOI: 10.1002/pssb.2221860135.

23 C. Lü and B. Yang, J. Mater. Chem., 2009, 19, 2884-2901.
24 M. K. Mishra, S. Kundu and G. De, J. Mater. Chem. C, 2016, 4, 1665-1674.

25 A. Tiwari and S. J. Dhobleb, RSC Adv., 2016, 6, 64400-64420.

26 D. P. Birnie III and N. J. Bendzko, Mater. Chem. Phys., 1999, 59, 26-35.

27 K. C. Pande and R. C. Mehrot, Z. Anorg. Allg. Chem., 1957, 290, 95-100.

28 R. Parra, M. S. Góes, M. S. Castro, E. Longo, P. R. Bueno and J. A. Varela, Chem. Mater., 2008, 20, 143-150.

29 D. P. Birnie, J. Mater. Sci., 2000, 35, 367-374.

30 C. J. Brinker and G. W. Scherer, The Physics and Chemistry of sol-gel processing, Academic press, 1990.

31 C. Lua and B. Yang, J. Mater. Chem., 2009, 19, 2884-2901.

32 R. Urlaub, et al., J. Non-Cryst. Solids, 2000, 265, 276-284.

33 K. Nakamoto, Infrared and Raman Spectra of Inorganic and Coordination Compounds, Wiley, New York, 3rd edn, 1978. 